Drug Delivery

\title{
Nanostructured Lipid Carriers-Containing Anticancer Compounds: Preparation, Characterization, and Cytotoxicity Studies
}

\section{Maria Luisa Bondì, Emanuela Fabiola Craparo, Gaetano Giammona, Melchiorre Cervello, Antonina Azzolina, Patrizia Diana, Anna Martorana \& Girolamo Cirrincione}

To cite this article: Maria Luisa Bondì, Emanuela Fabiola Craparo, Gaetano Giammona, Melchiorre Cervello, Antonina Azzolina, Patrizia Diana, Anna Martorana \& Girolamo Cirrincione (2007) Nanostructured Lipid Carriers-Containing Anticancer Compounds: Preparation, Characterization, and Cytotoxicity Studies, Drug Delivery, 14:2, 61-67, DOI: 10.1080/10717540600739914

To link to this article: https://doi.org/10.1080/10717540600739914

曲 Published online: 10 Oct 2008.

Submit your article to this journal $₫$

Џll Article views: 359

4 Citing articles: 33 View citing articles 


\title{
Nanostructured Lipid Carriers-Containing Anticancer Compounds: Preparation, Characterization, and Cytotoxicity Studies
}

\author{
Maria Luisa Bondì \\ Istituto per lo Studio dei Materiali Nanostrutturati, sez. di Palermo, Consiglio Nazionale delle Ricerche, \\ Palermo, Italy
}

\section{Emanuela Fabiola Craparo and Gaetano Giammona}

Dipartimento di Chimica e Tecnologie Farmaceutiche, Università di Palermo, Palermo, Italy

Melchiorre Cervello and Antonina Azzolina

Istituto di Biomedicina e Immunologia Molecolare “Alberto Monroy," Consiglio Nazionale delle

Ricerche, Palermo, Italy

\author{
Patrizia Diana, Anna Martorana, and Girolamo Cirrincione \\ Dipartimento Farmacochimico, Tossicologico e Biologico, Università di Palermo, Palermo, Italy
}

This article describes the development of nanostructured lipid carriers (NLC) as colloidal carriers for two antitumor compounds that possess a remarkable antineoplastic activity. But their limited stability and low solubility in water could give a very low parenteral bioavailability. Results revealed an enhancement of the cytotoxicity effect of drug-loaded NLC on human prostate cancer (PC-3) and human hepatocellular carcinoma (HuH-6, HuH-7) cell lines with respect to that of both free drugs. Results of characterization studies strongly support the potential application of these drugs-loaded NLC as prolonged delivery systems for lipophilic drugs by several administration routes, in particular for intravenous administration.

\section{Keywords Antitumor Drugs, Controlled Release, Human Hepatocel- lular Carcinoma Cells, Human Prostate Carcinoma Cells, Nanostructured Lipid Carrier.}

Colloidal carriers offer a great number of potential advantages as drug delivery systems, such as an improvement of bioavailability of poorly water-soluble drugs, resulting in an increased drug half life that reflects in an enhanced therapeutic performance (1). Great attention currently is paid to lipid particles with submicron size, the so-called solid lipid nanoparticles (SLN),

Received 21 November 2005; accepted 10 February 2006.

Address correspondence to Dr. Maria Luisa Bondì, Istituto per lo Studio dei Materiali Nanostrutturati, sez. di Palermo, Consiglio Nazionale delle Ricerche, Via Ugo la Malfa 153, 90146 Palermo, Italy. E-mail: marialuisa.bondi@ismn.cnr.it due to their biodegradability and ability to entrap a great variety of biologically active compounds $(2,3)$. SLN combine the advantages of other innovative colloidal carrier systems, such as liposomes and polymeric nanoparticles (e.g., physical stability, protection of incorporated labile compounds from degradation, controlled release of drug) while at the same time minimize their associate problems as toxicity of materials and difficulties in upscaling (4-6). More recently, modification of SLN, the so-called nanostructured lipid carriers (NLC), has been reported $(7,8)$. Such modifications overcome limitations of conventional SLN, such as unexpected dynamics of polymorphic transitions and inherently low drug incorporation capacity due to the crystalline structure of the solid lipid $(9,10)$. NLC can be realized by mixing spatially different lipids or liquid lipids (oils) with solid lipids. In the latter case, if a proper amount of oil is mixed with the solid lipid, a phase separation and the formation of oily nanocompartments within the solid lipid matrix can occur $(11,12)$. This leads to the possibility of incorporating a high amount of drug inside these systems, because liquid lipids solubilize drugs to a much higher extent than solid lipids (13). Moreover, control of drug release can be obtained due to encapsulating solid lipid (13).

8-cyano-6-phenyl-7-methyl-3-(para-chlorophenyl)-[2,1-d]pyrrolo-[1,2,3,5]tetrazine-4-one (compound A) and 8-cyano3,6-diphenyl-7-methyl-[2,1-d]-pyrrolo-[1,2,3,5]tetrazine-4-one (compound B), compounds that hold the deaza skeleton of the antitumor drug temozolomide ${ }^{\circledR}$, were synthesized (14). Such compounds were tested at the National Cancer Institute of Bethesda against 60 human cell lines that have grouped in disease subpanels including leukemia, nonsmall-cell lung, colon, 
central nervous system, melanoma, ovarian, renal, prostate, and breast tumors or cell lines. Details of this in vitro test system and the information, encoded by the activity pattern over all cell lines, have been reported previously (15-17). The antitumor activity of test compounds is given by three parameters for each cell line; $\mathrm{pGI}_{50}$ value $\left(\mathrm{GI}_{50}\right.$ is the molar concentration of the compound that inhibits 50\% net cell growth); pTGI (TGI is the molar concentration of the compound leading to total inhibition of the net cell growth); and $\mathrm{pLC}_{50}$ value $\left(\mathrm{LC}_{50}\right.$ is the molar concentration of the compound that induces $50 \%$ net cell death). Moreover, a mean graph midpoint (MG_MID) is calculated for each of the mentioned parameters, giving an average activity parameter over all cell lines. For the calculation of the MG_MID, insensitive cell lines are included with the highest concentration tested.

Both pyrrolotetrazinones were shown to be active against all the human cell lines tested with $\mathrm{GI}_{50}$ values at micromolar or submicromolar concentrations and, in the case of compound $\mathrm{A}$, even at nanomolar level (18). Compounds A and B possess extremely hydrophobic behavior, and for this reason their use in conventional dosage forms could be limited.

Compound $\mathrm{B}$ was evaluated as anticancer agent in an in vivo animal model in which polyvinylidene fluoride (PVDF) hollow fibers containing various human cancer cell cultures were implanted intraperitoneally (ip) and subcutaneously (sc) into mice (19). The effects of the compound on reduction of viable cancer cell mass compared with those of controls were determined. Compound B was tested in the hollow fibre assay against a panel of 12 human cell lines (non-small cell lung cancer: NCI-H23, NCI-H522; colon cancer: SW-620, COLO-205; CNS cancer: U251, SF-295; melanoma: LOX IMVI, UACC-62; ovarian cancer: OVCAR-3, OVCAR-5; breast cancer: MDAMB-231, MDA-MB-435), as described previously $(19,20)$. The compound was solubilized in 10\% DMSO in saline/Tween-80R and administrated ip once daily for a total of 4 doses at each of two dose levels ( 150 and $100 \mathrm{mg} / \mathrm{Kg}$ ). The day after the administration of the last compound dose, the fibers were collected and assessed for viable cell mass. Unfortunately, compound B was inactive under the conditions of test, in spite of the fact that in the in vitro tests against the same cell lines, it showed remarkable antiproliferative activity $\left(\mathrm{GI}_{50} 1.47 \cdot 10^{-5}-1.77 \cdot 10^{-7}\right)(18)$. Probably it decomposed before reaching the target.

Thus the formulation of pyrrolotetrazinones as drug delivery systems can be advantageous, considering that nanoparticulate systems such as NLC have been proposed for administration of drugs in cancer therapy. In fact, a critical advantage in treating tumors with nanoparticulate systems comes from the unique pathophysiological characteristics of solid tumors: extensive angiogenesis and hence hypervascularization, coupled with poor lymphatic drainage, which allow a facilitate extravasation into the tumor and an enhanced permeation and retention effect (EPR effect) of colloidal systems (21-24). For this reason, nanoparticles such as NLC are intriguing since they are able to increase drug levels within the tumor by circumventing the tumor vascu- lature, to decrease normal tissue toxicities by reducing the level of drug in the systemic circulation, and to act as depot for the extended release of drug (21). However, the accumulation of lipid nanoparticles within the Kuppfer cells of the liver generally is found after intravenously injection. With the exception of liver diseases like hepatic neoplasms and liver infections, passive liver targeting can be easily avoided by modulating their physicochemical characteristics as particle size, surface charge, and surface hydrophobicity $(7,25)$.

Our article describes the development of NLC loaded with compounds A and B as potential carriers for drug administration in cancer treatment. The preparation and the characterization of empty and drug-loaded NLC regarding loading capacity, size, shape and zeta potential values are described. Drug release studies in human plasma and viability assays against three human cancer cell lines also were evaluated.

\section{MATERIALS AND METHODS}

\section{Materials}

8-cyano-6-phenyl-7-methyl-3-(para-chlorophenyl)-[2,1-d]pyrrolo-[1,2,3,5]tetrazine-4-one (compound A) and 8-cyano3,6-diphenyl-7-methyl-[2,1-d]-pyrrolo-[1,2,3,5]tetrazine-4-one (compound B) were prepared according to the procedure reported in literature [14] by reacting 3-cyano-4-methyl-5-phenyl2-diazopyrrole with 4-chloro-phenyl- and phenyl-isocyanate for compounds $\mathrm{A}$ and $\mathrm{B}$, respectively: to a solution of the diazo compound $(2 \mathrm{mmol})$ in dry dimethylformamide $(10 \mathrm{ml})$, the suitable isocyanate $(20 \mathrm{mmol})$ in dry dimethylformamide $(10 \mathrm{ml})$ was added dropwise, at room temperature, in the dark. The reaction mixture was stirred until the diazo band at $\sim 2110 \mathrm{~cm}^{-1}$ disappeared (24-72 hr), then poured onto crushed ice. The solid precipitate was filtered off, air dried, and purified by flash chromatography with dichloromethane as eluant to give the desired compounds.

Compritol 888 ATO (mixture of approximately mono-, diand triglycerides of behenic acid, at 15,50 and $35 \% \mathrm{w} / \mathrm{w}$, respectively) was a gift of Gattefossé s.a. (Saint-Priest, France). Miglyol 812 (caprylic/capric triglycerides, medium chain triglyceride) was supplied by Sasol GmbH (Germany). Soy phosphatidylcholine 95\% (Epikuron 200) was donated by Degussa Texturant Systems (Germany). Taurocholic acid sodium salt was purchased from Sigma-Aldrich (Milan, Italy). The other chemicals were of obtained from Sigma and were of analytical grade.

\section{Preparation of Empty NLC}

NLC were prepared through the precipitation technique. The lipid phase was prepared by melting Compritol 888 ATO $(150 \mathrm{mg})$ at $85^{\circ} \mathrm{C}$ and then adding Miglyol $812(30 \mathrm{mg})$. Epikuron $200(36 \mathrm{mg})$ was solubilized in ethanol $(2 \mathrm{ml})$ at $85^{\circ} \mathrm{C}$ and then the melted lipid phase was added under stirring. NLC were obtained by dispersing the obtained warm solution in cold water at $2-3^{\circ} \mathrm{C}(100 \mathrm{ml})$ containing taurocholate sodium salt 
(130.7 mg), by using an Ultra Turrax T25 (IKA, D-Staufen). The obtained NLC dispersion was submitted to exhaustive dialysis by using Visking Tubing Dialysis 18/32" (with a molecular weight cut-off of 12,000-14,000). The NLC dispersion were freeze-dried using a Modulyo freeze-dryer (Edwards, Crawley, UK).

\section{Preparation of Drug-Loaded NLC}

The lipid phase was prepared by heating Compritol 888 ATO $(150 \mathrm{mg})$ at $85^{\circ} \mathrm{C}$ and adding Miglyol $812(30 \mathrm{mg})$. Epikuron $200(36 \mathrm{mg})$ was solubilized in ethanol $(2 \mathrm{ml})$ at $85^{\circ} \mathrm{C}$ and then the melted lipid phase was added under stirring. The compound (A or B) was added to the hot solution and the solution was left for 10 min under mechanical stirring. A-loaded NLC and B-loaded NLC were obtained by dispersing the obtained warm solution in cold water at $2-3^{\circ} \mathrm{C}(100 \mathrm{ml})$ containing taurocholate sodium salt $(130.7 \mathrm{mg})$, by using an Ultra Turrax. The drugloaded NLC dispersions were submitted to exhaustive dialysis and then freeze-dried.

\section{Characterization of Empty and Drug-Loaded NLC Particle Size Determination}

The average diameter and polydispersity index of empty and drug-loaded NLC were determined by photon correlation spectroscopy (PCS) using a Zetasizer Nano ZS (Malvern Instrument, Herrenberg, Germany) that utilizes the Non-Invasive Back-Scattering (NIBS) technique. Each sample was appropriately diluted with filtered water $(0.2 \mu \mathrm{m})$ and the reading was carried at a $173^{\circ}$ angle in respect to the incident beam. Each reported value was the average of three measurements.

\section{Zeta Potential Measurements}

The zeta potential values were measured using principles of laser doppler velocimetry and phase analysis light scattering (M3-PALS technique). For this purpose, a Zetasizer Nano ZS Malvern Instrument equipped with a He-Ne laser at a power $\mathrm{P}=4.0 \mathrm{~mW}$ and with $\lambda=633 \mathrm{~nm}$ was used (Herrenberg, Germany). Empty and drug-loaded NLC samples were dispersed in filtered $(0.2 \mu \mathrm{m})$ double-distilled water, in an aqueous solution of $\mathrm{NaCl}(0.9 \% \mathrm{w} / \mathrm{v})$ or in phosphate buffer at $\mathrm{pH}$ 7.4. Each sample was analyzed in triplicate.

\section{HPLC Analysis of Compounds A and B}

An adequate HPLC method was developed to study drug loading and release process of A and B. The HPLC analysis was performed at room temperature using a Shimatzu instrument equipped with a reversed-phase $\mathrm{C}_{18}$ column ( $\mu$ Bondapak, $3 \mu \mathrm{m}, 150 \times 4.6 \mathrm{~mm}$ i.d., Supelco). A mixture of $\mathrm{CH}_{3} \mathrm{OH}$ and $\mathrm{H}_{2} \mathrm{O}(80: 20 \mathrm{v} / \mathrm{v})$ with a flow rate of $0.4 \mathrm{ml} \mathrm{min}^{-1}$ was used as mobile phase. The HPLC column system was connected with a UV-Vis detector (Shimatzu). A and B, either fresh or released from NLC, were injected and measured at the wavelength of $360 \mathrm{~nm}$.
Quantification of each compound was done by using a calibration curve obtained using solutions at known drug concentrations. The straight-line equation for compound A was:

$$
\begin{aligned}
& y=58380 x-136292(y=\text { peak area; } \\
& \left.x=\text { drug concentration } \mu \mathrm{gml}^{-1}\right)
\end{aligned}
$$

The linear regression value was $r=0,9984$. The linearity of the method was studied in the range $25-100 \mu \mathrm{g} \mathrm{ml}^{-1}$.

The straight-line equation for compound $\mathrm{B}$ was:

$$
\begin{aligned}
& y=51437 x-108612(y=\text { peak area } \\
& \left.x=\text { drug concentration } \mu \mathrm{gml}^{-1}\right)
\end{aligned}
$$

The linear regression value was $r=0,9996$. The linearity of the method was studied in the range $25-100 \mu \mathrm{g} \mathrm{ml}^{-1}$.

\section{Determination of Loading Capacity}

The loading capacity of NLC, expressed as amount of loaded drug in percent related to the lipid phase (matrix lipid + drug), was calculated using the following procedure. Typically, $5 \mathrm{mg}$ of each batch of freeze-dried drug-loaded NLC were solubilized in $5 \mathrm{ml}$ of dichloromethane. The organic solution was filtered through $0.45 \mu \mathrm{m}$ (PTFE membrane) filters and analyzed by HPLC to determine the amount of compounds.

\section{Release of $A$ and $B$ from NLC in Human Plasma}

Release of A and B from NLC samples was assayed at 7 prefixed time intervals. Seven suspensions of each batch containing $10 \mathrm{mg}$ of drug-loaded NLC in $5 \mathrm{ml}$ of human plasma were prepared and kept at $37^{\circ} \mathrm{C} \pm 0.1^{\circ} \mathrm{C}$ under mechanical stirring. At fixed time intervals, each sample was centrifuged at $13000 \mathrm{~g}$ for $10 \mathrm{~min}$ at $4^{\circ} \mathrm{C}$. Then 2-propanol $(4 \mathrm{ml})$ was added to $1 \mathrm{ml}$ of supernatant and the obtained suspension was centrifuged at $13000 \mathrm{~g}$ for $5 \mathrm{~min}$ at $4^{\circ} \mathrm{C}$. Then supernatant was assayed by HPLC.

\section{Cell Viability Assays}

All experiments were conducted using human hepatocellular carcinoma (HuH-6, HuH-7) cells and human prostate cancer (PC-3) cells. HuH-6 and HuH-7 cell lines were grown as a monolayer in RPMI 1640 medium (Sigma, Milan, Italy) supplemented with $10 \%$ heat-inactivated fetal calf serum (Invitrogen, Milan, Italy), $2 \mathrm{mmol} / \mathrm{l} \mathrm{L}$-glutamine, $1 \mathrm{mmol} / \mathrm{l}$ sodium pyruvate, and penicillin/streptomycin solution (100 units/ml) (all from Sigma), at $37^{\circ} \mathrm{C}$ in $5 \% \mathrm{CO}_{2}$ humidified atmosphere. They were subcultured after trypsinization (with $0.05 \%$ trypsin/0.02\% EDTA in PBS, from Sigma) for $5 \mathrm{~min}$ at room temperature, washing and suspended again in complete medium. The human prostate PC-3 cell line was maintained as above unless that sodium pyruvate was omitted.

Cells in complete medium were seeded on 96-well plates at a density of 5,000 per well and allowed to adhere overnight. Before 


\section{TABLE 1}

Dimensional analysis by photon correlation spectroscopy and loading capacity of A- and B-loaded NLC prepared by precipitation method.

\begin{tabular}{lccr}
\hline & Size $(\mathrm{nm})$ & $\begin{array}{c}\text { Polydispersity } \\
\text { Index }\end{array}$ & $\begin{array}{c}\text { Loading capacity } \\
(\% \mathrm{w} / \mathrm{w})\end{array}$ \\
\hline Empty NLC & $116.1 \pm 0.7$ & $0.240 \pm 0.005$ & - \\
$\boldsymbol{A}$-loaded NLC & $122.3 \pm 0.9$ & $0.148 \pm 0.030$ & $21.0 \pm 0.13$ \\
B-loaded NLC & $126.2 \pm 1.1$ & $0.172 \pm 0.011$ & $19.2 \pm 0.11$ \\
\hline
\end{tabular}

Each value is the mean of three experiments \pm S.E.

use, empty or drug-loaded NLC suspension in double distilled water $(1 \mathrm{mg} / \mathrm{ml})$ was freeze-dried. Then, freeze-dried empty and drug-loaded NLC samples were dispersed in the proper medium using a water bath sonication for 10 min (Transsonic 310, Elma, Germany).

At time 0 , the cell medium was replaced with fresh complete medium, and various concentrations $(0.1-5 \mu \mathrm{M})$ of A or B, free in DMSO solution or blended in NLC, were added and cells were cultured for $72 \mathrm{hrs}$. Unloaded NLC toxicity also was evaluated on the three cell lines growth at the highest concentrations corresponding to that of $5 \mu \mathrm{M}$ of A- or B-loaded NLC. At the end of incubation, MTT assay was performed using the CellTiter Aqueous OneSolution kit (Promega Corporation, Madison, WI, USA) containing 3-(4,5-dimethylthiazol-2-yl)-5-(3-carboxymethoxyphenyl)-2(4-sulphophenyl)-2H-tetrazolium (MTT) and phenazine ethosulfate. Briefly, $15 \mu \mathrm{l}$ of the above mentioned commercial solution were added to each well. The plates were incubated for $1 \mathrm{~h}$ in a humidified atmosphere at $37^{\circ} \mathrm{C}$ in $5 \% \mathrm{CO}_{2}$. The bioreduction of the MTT dye was assessed by measuring the absorbance of each well at $490 \mathrm{~nm}$. Cytotoxicity was expressed as a percentage of the absorbance measured in the control cells $(100 \%)$. Experiments were performed in triplicate, with the results reflecting the mean and standard deviation of the triplicate of each group.

\section{RESULTS AND DISCUSSION}

In our study, nanostructured lipid carriers (NLC) empty and loaded with two antitumor compounds (A and B) were pre- pared successfully by the precipitation technique. In these systems, solid and liquid lipids were blended to form a solid matrix that contains liquid oily nanocompartments to accommodate the drugs.

\section{Drug Loading and Size Analysis}

These systems were characterized in terms of drug loading capacity and particle size distribution to investigate whether these possess the best characteristics to be used as a colloidal pharmaceutical formulation for parenteral administration of drugs. In Table 1 , loading capacity (\% w/w), size ( $\mathrm{nm})$, and polydispersity index (P.I.) are reported. The data indicate that the average diameter of empty and drug-loaded NLC batches were quite small and in the range of 110 to $130 \mathrm{~nm}$, with the empty system smaller than drug-loaded NLC. This result probably is due to a higher amount of lipid phase, containing also the drug, precipitated in the aqueous cold phase for obtaining drug-loaded NLC. The quite low P.I. values also indicate a good dimensional homogeneity of the prepared systems. B-loaded NLC were found to be the largest (about $126 \mathrm{~nm}$ ) since the amount of entrapped compound is lower than A-loaded one.

However, the loading capacity, determined by HPLC analysis and expressed as the percentage ratio between the weight of compound entrapped and the total weight of NLC, results are very high in both the A- and B-loaded samples, at 21.0 and $19.2 \% \mathrm{w} / \mathrm{w}$, respectively. These results appear important because these characteristics after the possibility of administering these systems loaded with a considerable amount of drug due to small size by all the possible routes.

TABLE 2

Zeta potential values $(\mathrm{mV})$ of empty and drug-loaded NLC

\begin{tabular}{lccr}
\hline & $\mathrm{H}_{2} \mathbf{O}$ & $\mathrm{NaCl}$ & $\mathrm{PBS}(\mathrm{pH} 7.4)$ \\
\hline Empty NLC & $-32.22 \pm 1.0$ & $-11.19 \pm 0.7$ & $-14.51 \pm 1.7$ \\
A-loaded NLC & $-33.57 \pm 1.8$ & $-9.11 \pm 1.8$ & $-13.26 \pm 1.5$ \\
B-loaded NLC & $-31.07 \pm 0.3$ & $-8.11 \pm 0.6$ & $-11.73 \pm 1.1$ \\
\hline
\end{tabular}

Each value is the mean of three experiments \pm S.E.

$\mathrm{H}_{2} \mathrm{O}=$ Double-distilled water, $\mathrm{NaCl}=$ sodium chloride.

$\mathrm{PBS}=$ phosphate buffered solution . 


\section{Zeta Potential Measurements}

To investigate the surface charge of empty and drug-loaded NLC, zeta potential measurements were carried out in three different aqueous media. Table 2 shows the zeta potential values of empty and drug-loaded NLC batches.

The surface charge values are negative for both empty and drug-loaded samples in all the investigated media. Moreover, these values are higher in double-distilled water ranging between -31 and $-34 \mathrm{mV}$. The presence of electrolytes causes a diminution of surface charge of all the investigated samples. However, using the same dispersing medium, no statistically significant differences were found between various examined batches. The presence of a charge onto the surface is important because it makes these systems more stable when dispersed into an aqueous solution, reducing the occurrence of the aggregation phenomenon.

\section{Release Studies in Human Plasma}

To evaluate the controlled release potentiality of the investigated NLC, in vitro release studies were carried out in human plasma. Figure 1 shows the percentage amount of compound (A or B) released from NLC samples as function of incubation time.

Experimental results for both A and B compounds showed an initial low burst effect (11\% and 18\%, respectively, of compound released after 15 min of incubation) and a release considerably slow during the investigation time. In fact, less than $30 \%$ and $38 \%$ of A and B, respectively, were released from NLC within $24 \mathrm{hr}$ incubation. After $24 \mathrm{hr}$ of incubation loaded samples were analyzed and both unreleased compounds were found into nanoparticles. This fact is very important considering that loaded NLC will release in the bloodstream a low percentage of loaded compounds that could reach the target site (as a tumor)

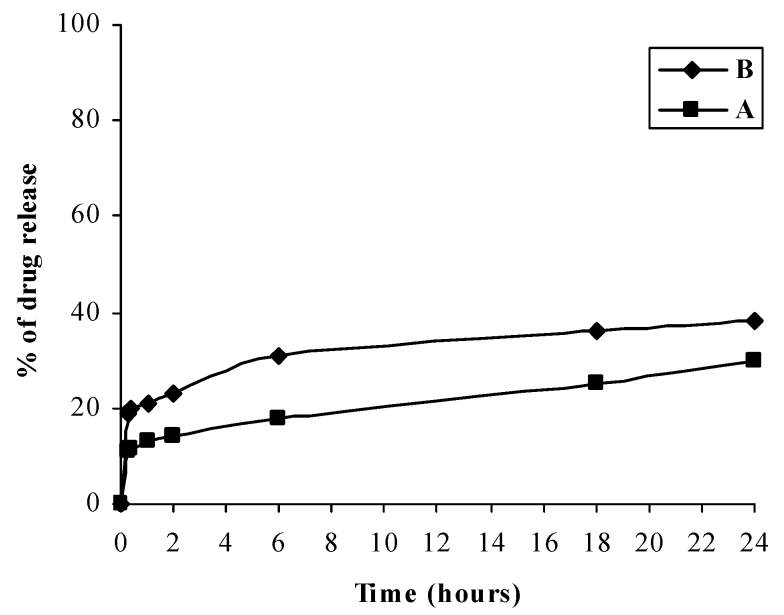

FIG. 1. Release profiles of A ( $\mathbf{\square})$ and B ( $\bullet$ from drug-loaded NLC in human plasma. Each value is the mean of three experiments. All calculated standard devintions were less than $3 \%$ of the mean values and thus not reported.
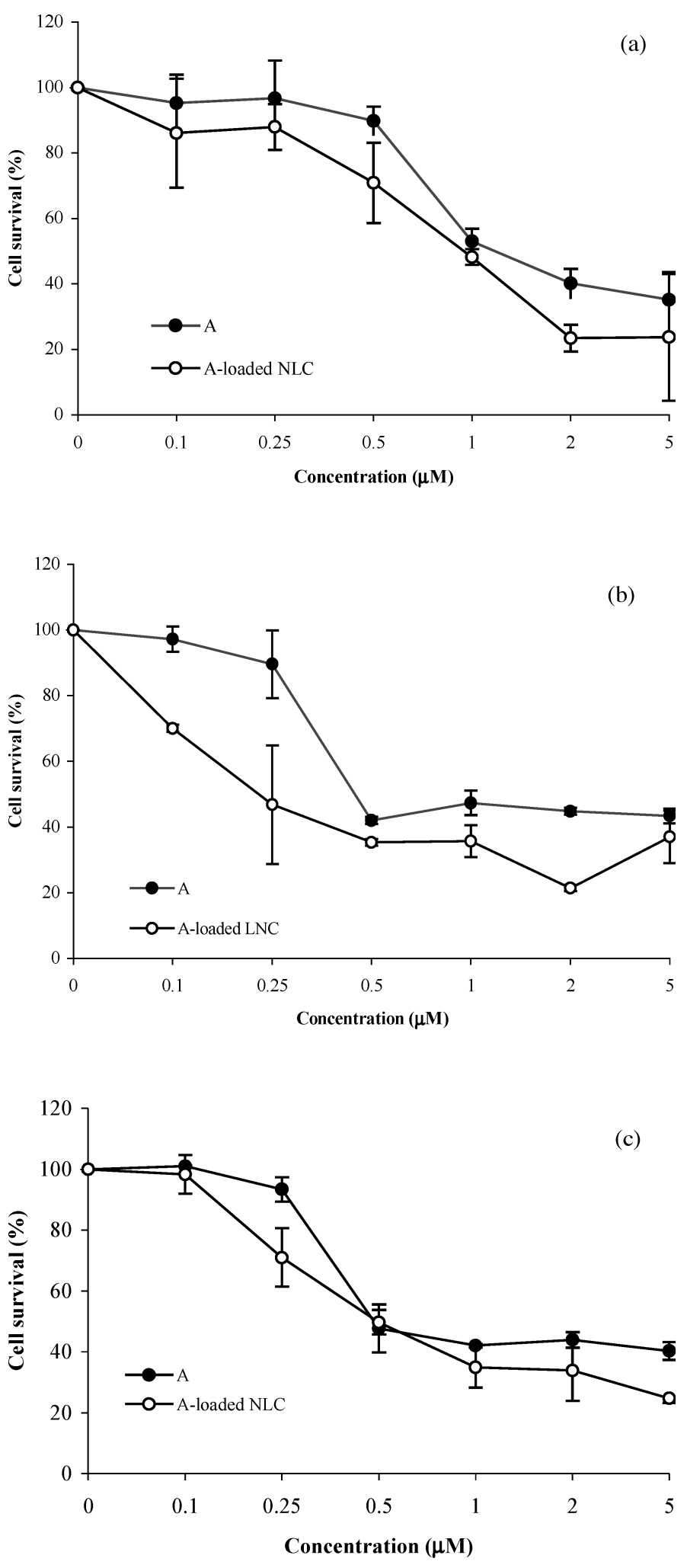

FIG. 2. Effects of A (•) and A-loaded NLC ( $)$ on the viability of HuH-6 (a), HuH-7 (b), and PC-3 (c) cells. Cells were grown and treated for $72 \mathrm{hr}$. Experiments were performed in triplicate, with the results showing the mean and standard deviation of the triplicate of each group. The experiments were repeated three times with similar results. 
inside of the lipid carrier and there it could be released. These systems, which possess surface and dimensional characteristics useful for parenteral administration, are able to give a prolonged release of compounds A and B.

\section{Viability Assays}

To evaluate the activity of compounds $\mathrm{A}$ and $\mathrm{B}$ when administered as free forms or loaded in NLC, in vitro cytotoxicity assays were carried out on human hepatocellular carcinoma (HuH-6, $\mathrm{HuH}-7$ ) and on human prostate cancer (PC-3) cell lines by using the MTT test. To ensure that the cytotoxicity was caused by the compound itself and not by formulation components of NLC, cells were incubated with unloaded nanoparticles. No cytotoxicity of the unloaded NLC was observed after $72 \mathrm{hr}$ on the three cell lines studied, even at the highest concentration (not shown), indicating the absence of cytotoxicity of empty NLC or any material released from them at the conditions used for experiments. Figure 2 shows the survival curves of HuH-6 (Figure 2A), HuH-7 (Figure 2B), and PC-3 (Figure 2C) cells after exposure to free $\mathrm{A}$ and $\mathrm{A}-\mathrm{B}$-oaded NLC. The effect of $\mathrm{B}$ and B-loaded NLC was tested only on HuH-6 cells and reported in Figure 3.

The data indicate that the cytotoxicity of compounds, both in the free form and blended in NLC, on all the cell lines tested increase in a dose-dependent manner in the presence of either A or B. Moreover, the incorporation of compounds into the lipid carrier seems to enhance the cytotoxicity effect of both compounds on tested cells. This fact is very important since these compounds are not freely soluble in water, and the result can be explained by incorporation within a lipid carrier probably enhances the penetration of compounds inside the cell by processes such as

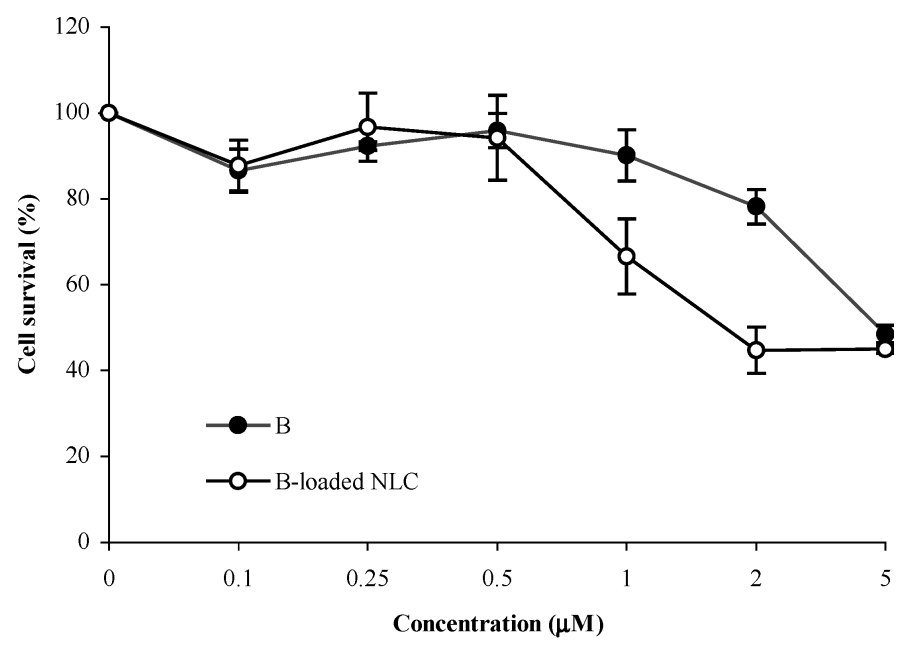

FIG. 3. Effects of B (•) and B-loaded NLC (०) on the viability of HuH-6 cells. Cells were grown and treated for $72 \mathrm{hr}$. Experiments were performed in triplicate, with the results showing the mean and standard deviation of the triplicate of each group. The experiments were repeated three times with similar results. endocytosis. Moreover, nanoparticles once in the bloodstream are mostly recognized by macrophages of the liver and other organs of the reticuloendotelial system, making these systems potentially useful for drug passive targeting for the treatment of hepatic neoplasms.

\section{CONCLUSION}

In this study the utility of NLC dispersions as carriers for parenteral delivery of antitumor drugs was explored. NLC, constituted of bioacceptable lipids, were prepared by the precipitation technique as empty systems or drug-loaded with two antitumoral pyrrolotetrazinones, A and B. Empty and drug-loaded NLC showed average diameters in the colloidal size range, a spherical shape, a good loading capacity, and a prolonged drug release of both compounds in human plasma. In vitro cytotoxicity assays on human hepatocellular carcinoma (HuH-6 and $\mathrm{HuH}-7$ ) and on human prostate cancer (PC-3) cell lines have shown that the incorporation of compounds A and B into the lipid carrier enhance the cytotoxicity effect of both compounds on tested cells.

Results of characterization strongly support the potential application of both A- and B-loaded NLC as a drug delivery system for controlled release by several administration routes.

\section{REFERENCES}

1. Miura, H., Onishi, H., Sasatsu, M., and Machida, Y. 2004. Antitumor characteristics of methoxypolyethylene glycol-poly(DL-lactic acid) nanoparticles containing camptothecin. J. Control. Rel. 97:101-113.

2. Mehnert, W., and Mader, K. 2001. Solid lipid nanoparticles. Production, characterization and applications. Adv. Drug Del. Rev. 47:165-196.

3. Muller, R. H., Mader, K., and Gohla, S. 2000. Solid lipid nanoparticles (SLN) for controlled drug delivery - a review of the state of the art. Eur. J. Pharm. Biopharm. 50:161-177.

4. Lasic, D. D. 1998. Novel applications of liposomes. Tibtech 16:307-321.

5. Heath, T. H. 1988. Liposomes dependent drugs. In Liposomes as Drug Carriers: Trends and Progress. ed. Gregoriadis, G., San Francisco: Wiley. pp. 709-718.

6. Smith, A., and Hunneyball, I. M. 1986. Evaluation of polylactid as a biodegradabledrug delivery system for parenteral administration. Int. J. Pharm. 30:215-230.

7. Wissing, S. A., Kayser, O., and Muller, R. H. 2004. Solid lipid nanoparticles for parenteral drug delivery. Adv. Drug Del. Rev. 56:1257-1272.

8. Muller, R. H., Radtke, M., and Wissing, S. A., 2002. Solid lipid nanoparticles (SLN) and nanostructured lipid carriers (NLC) in cosmetic and dermatological preparations. Adv. Drug Del. Rev. 54(1):S131-S155.

9. Muller, R. H., Radtke, M., and Wissing, S. A. 2002. Nanostructured lipid matrices for improver microencapsulation of drugs. Int. J. Pharm. 242:121128.

10. Radtke, M., and Muller, R. H. 2001. NLC-nanostructured lipid carriers: the new generation of lipid drug carriers. New Drugs 2:97-138.

11. Jenning, V., Thunemann, A. F., and Gohla, S. H. 2000. Characterization of a novel solid lipid nanoparticle carrier system based on binary mixtures of liquid and solid lipids. Int. J. Pharm. 199:167-177.

12. Jenning, V., and Gohla, S., 2001. Encapsulation of retinoids in solid lipid nanoparticles (SLN). J. Microencaps. 18:149-158.

13. Jores, K., Mehnert, W., Drechsler, M., et al. 2004. Investigation on the structure of solid lipid nanoparticles (SLN) and oil-loaded solid lipid nanoparticles by photon correlation spectroscopy, field-flow fractionation and transmission electron microscopy. J. Control. Rel. 95:217-227. 
14. Diana, P., Barraja, P., Lauria, A., et al. 1999. Pyrrolo[2,1-d][1,2,3,5]tetrazines, a new class of azolotetrazines related to the antitumor drug temozolomide. Synthesis 2082.

15. Grever, M. R., Sherpartz, S. A., and Chabner, B. A. 1992. The National Cancer Institute: cancer drug discovery and development program. Semin. Oncol. 19:622-638.

16. Monks, A. P., Scudiero, D. A., Skehan, P., et al. 1991. Feasibility of a high-flux anticancer drug screen utilizing a derived panel of human tumor cell lines in culture. J. Natl. Cancer Inst. 83:757766 .

17. Weinstein, J. N., Meyers, T. G., O'Connor, P. M., et al. 1997. An information-intensive approach to the molecular pharmacology of cancer. Science 275:343-349.

18. Diana, P., Barraja, P., Lauria, A., et al. 2003. Pyrrolo[2,1-d][1,2,3,5]tetrazine-4(3H)-ones, a new class of azolotetrazines with potent antitumor activity. Bioorg. Med. Chem. 11:2371-2380.

19. Plowman, J., Dykes, D. J., Hollingshead, M., et al., 1997. In Anticancer Drug Development Guide: Preclinical Screening, Clinical Trials, and Approval; ed. Teicher B., Totowa, NJ: Humana Press. 101125.

20. Hollingshead, M., Plowman, J., Alley, M. C., et al., 1999. The hollow fiber assay. Contrib. Oncol. 54:109-120.

21. Brigger, I., Dubernet, C., and Couvreur, P. 2002. Nanoparticles in cancer therapy and diagnosis. Adv. Drug Del. Rev. 54:631-651.

22. Maeda, H., Wu, J., Sawa, T., et al. 2000. Tumor vascular permeability and the EPR effect in macromolecular therapeutics: a review. J. Control. Rel. 65:271-284.

23. Maeda, H. 2001. The enhanced permeability and retention (EPR) effect in tumor vasculature: the key role of tumor-selective macromolecular drug targeting. Adv. Enzyme Regul. 41:189-207.

24. Noguchi, Y., Wu, J., Duncan, R., et al. 1998. Early phase tumor accumulation of macromolecules: a great difference in clearance rate between tumor and normal tissues. Jpn. J. Cancer Res. 89:307-314.

25. Scholer, N., Hahn, H., Muller, R. H., and Liesenfeld, O. 2002. Effect of lipid matrix and size of solid lipid nanoparticles (SLN) on the viability and cytokine production of macrophages. Int. J. Pharm. 231:167-176. 